\title{
(6) OPEN ACCESS \\ Canakinumab reverses overexpression of inflammatory response genes in tumour necrosis factor receptor-associated periodic syndrome
}

\author{
Rebecca Torene, ${ }^{1}$ Nanguneri Nirmala, ${ }^{1}$ Laura Obici, ${ }^{2}$ Marco Cattalini, ${ }^{3}$ \\ Vincent Tormey, ${ }^{4}$ Roberta Caorsi, ${ }^{5}$ Sandrine Starck-Schwertz, ${ }^{6}$ Martin Letzkus, ${ }^{6}$ \\ Nicole Hartmann, ${ }^{6}$ Ken Abrams, ${ }^{7}$ Helen Lachmann, ${ }^{8}$ Marco Gattorno ${ }^{5}$
}

\begin{abstract}
Handling editor Tore K Kvien
- Additional material is published online only. To view please visit the journal online (http://dx.doi.org/10.1136/ annrheumdis-2016-209335).

${ }^{1}$ Novartis Institutes for Biomedical Research, Cambridge, Massachusetts, USA

${ }^{2}$ Amyloid Centre, IRCCS Policlinico San Matteo, Pavia, Italy

${ }^{3}$ Pediatric Clinic, University of Brescia and Spedali Civili, Brescia, Italy

${ }^{4}$ Galway University Hospitals, Galway, Ireland

${ }^{5} \mathrm{G}$ Gaslini Institute, Genova, Italy

${ }^{6}$ Novartis Institutes for Biomedical Research, Basel, Switzerland

${ }^{7}$ Novartis Pharmaceuticals, East Hanover, New Jersey, USA

${ }^{8}$ University College London Medical School, London, UK
\end{abstract}

Correspondence to Dr Marco Gattorno, UO Pediatria II, G. Gaslini Institute, Via Gaslini 5, Genova 16147, Italy; marcogattorno@ ospedale-gaslini.ge.it

Received 4 February 2016 Revised 14 June 2016 Accepted 20 June 2016 Published Online First 29 July 2016

\section{ABSTRACT}

Objective To explore whether gene expression profiling can identify a molecular mechanism for the clinical benefit of canakinumab treatment in patents with tumour necrosis factor receptor-associated periodic syndrome (TRAPS).

Methods Blood samples were collected from 20 patients with active TRAPS who received canakinumab $150 \mathrm{mg}$ every 4 weeks for 4 months in an open-label proof-of-concept phase II study, and from 20 agedmatched healthy volunteers. Gene expression levels were evaluated in whole blood samples by microarray analysis for arrays passing quality control checks.

Results Patients with TRAPS exhibited a gene expression signature in blood that differed from that in healthy volunteers. Upon treatment with canakinumab, many genes relevant to disease pathogenesis moved towards levels seen in the healthy volunteers.

Canakinumab downregulated the TRAPS-causing gene (TNF super family receptor 1A (TNFRSF1A)), the drugtarget gene (interleukin (IL)-1B) and other inflammationrelated genes (eg, MAPK 14). In addition, several inflammation-related pathways were evident among the differentially expressed genes. Canakinumab treatment reduced neutrophil counts, but the observed expression differences remained after correction for this.

Conclusions These gene expression data support a model in which canakinumab produces clinical benefit in TRAPS by increasing neutrophil apoptosis and reducing pro-inflammatory signals resulting from the inhibition of IL-1 $\beta$. Notably, treatment normalised the overexpression of TNFRSF1A, suggesting that canakinumab has a direct impact on the main pathogenic mechanism in TRAPS. Trial registration number NCT01242813.

\section{INTRODUCTION}

Tumour necrosis factor (TNF) receptor-associated periodic syndrome (TRAPS), an autosomaldominant disease, is one of a group of hereditary autoinflammatory periodic fever syndromes that includes familial Mediterranean fever, hyperimmunoglobulin D syndrome and the cryopyrinassociated periodic syndromes (CAPS), among others. $^{1} 2$ Mutations of the TNF super family receptor 1A (TNFRSF1A) gene are responsible for TRAPS, which is characterised by recurrent fever, myalgia, abdominal pain, rash, headaches, ocular symptoms and in some cases, amyloid A amyloidosis. $^{3-6}$ TRAPS, along with other autoinflammatory disorders, is associated with severe inflammation without a concomitant increase in autoantibodies or antigen-specific $\mathrm{T}$ cells. ${ }^{3}{ }^{7}$ Overexpression of interleukin (IL)- $1 \beta$ from circulating monocytes collected during disease flares has been recently described. ${ }^{8}$ IL- $1 \beta$ is recognised as a key pro-inflammatory cytokine in a variety of inflammatory conditions. ${ }^{9} 10$

Although the connection between TNFRSF1A mutation and increased release of IL-1 $\beta$ remains unclear, it is suspected that misfolding of the TNFR1 protein product triggers stress-related responses and accompanying inflammatory reactions including enhanced IL-1 $\beta$ release. ${ }^{1} 11 \quad 12$ TNFRSF1A mutations have also been suggested to affect pro-inflammatory signalling downstream of TNFR1, leading to constitutive activation of the

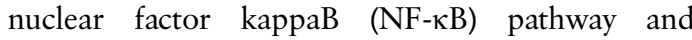
increased cytokine secretion; some mutations may also enhance IL-1 $\beta$ signalling due to the hyperinflammatory background in TRAPS. ${ }^{13}$ Given the molecular and clinical profiles of TRAPS, the IL-1 pathway has been hypothesised to be a viable therapeutic target, which has been supported by small case series demonstrating responses to anakinra. $^{14} 15$

Canakinumab is a high-affinity human monoclonal antihuman IL-1 $\beta$ antibody of the $\operatorname{IgG} 1 / \kappa$ isotype $^{16}{ }^{17}$ It is designed to bind to human IL-1 $\beta$, blocking the interaction of the cytokine with its receptor, and thus functionally neutralising its bioactivity without preventing binding of the natural endogenous inhibitor, IL-1 receptor antagonist, or IL- $1 \alpha$ to IL- 1 receptors. In a phase II proof-ofconcept study, canakinumab treatment provided complete or near-complete clinical responses in 19 of 20 patients with active recurrent or chronic TRAPS (ClinicalTrials.gov identifier number NCT01242813). ${ }^{18}$ We performed an analysis of gene expression from patients in this study and agematched healthy volunteers to characterise treatment-induced alterations.

\section{METHODS}

\section{Study design and patients}

The design of the open-label, multicentre, proof-ofconcept study is described separately (ClinicalTrials. gov identifier number NCT01242813). ${ }^{18}$ Briefly, 
patients 7 years and older with a genetically confirmed diagnosis of TRAPS and active recurrent or chronic disease received canakinumab $150 \mathrm{mg}$ subcutaneously every 4 weeks (q4wk) during a 4-month treatment period (days 1, 29, 57 and 85). A singledose up-titration to $300 \mathrm{mg}$ was permitted at day 8 in nonresponders. Upon completion of the treatment period on day 113, patients entered a treatment withdrawal/follow-up period lasting up to 5 months. Whole blood samples for microarray analysis of gene expression levels were collected at baseline, day 15 and day 113 from 20 patients in the study cohort, and on one occasion from 20 untreated age-matched healthy volunteers.

\section{Gene expression analyses}

Gene expression profiling was performed to identify differentially expressed genes between baseline samples and those collected during canakinumab treatment (day 15 and day 113) in patients with TRAPS and between the patients with TRAPS and the healthy volunteers. Blood samples from consenting patients were collected in PAXgene blood RNA tubes according to the manufacturer's guidelines (PreAnalytiX, Hombrechtikon, Switzerland) and stored at $-80^{\circ} \mathrm{C}$ until RNA extraction. The total RNA from whole blood was isolated with the PAXgene Blood RNA Kit (Qiagen, Hilden, Germany) according to the manufacturer's recommendations. The amplified cDNA was hybridised to Affymetrix Human Genome 133 Plus 2.0 arrays following standard procedures.

\section{Analytical methods}

The probe set annotation 'hgu133plus2hsentrezg' was obtained from http://brainarray.mbni.med.umich.edu/ and used for mapping of probe sets to genes. With use of this custom chip definition file, statistical analyses were performed on the gene level, as opposed to the probe level. All microarrays passing quality control (QC) were subjected to robust multi-array average condensing. The $2 \%$ trimmed mean of each chip was scaled to a target intensity of 150 . The analysis of differentially expressed genes is described in the online supplementary appendix.

\section{Pathway analysis}

Differentially expressed genes were mapped onto pathway maps using gene symbols in Metacore. We examined a number of canonical pathway maps from Metacore relevant to the pathogenesis of TRAPS including immune response toll-like receptor (TLR) signalling pathway, immune response IL-1 signalling pathway, apoptosis and survival: endoplasmic reticulum stress response pathway and the autophagy map. Genes that were upregulated or downregulated by at least 1.3 -fold were considered for this analysis.

\section{RESULTS \\ Identification of a TRAPS gene expression signature in untreated patients}

A total of 20 patients with TRAPS (mean age, 34.6 \pm 18.36 years; range, $7.0-77.8$ years; TNFRSF1A gene mutations shown in online supplementary table S1) entered the trial, of whom 19 patients had at least one microarray dataset that had passed QC. Similarly, 19 healthy controls had a microarray that passed QC. After condensing to gene level analysis, filtering Affymetrix control probes and removing low intensity probes, 6642 genes remained in the data set. The disease-causing gene TNFRSF1A was upregulated in patients with TRAPS by 1.4 -fold compared with the healthy volunteers. Other genes relevant to inflammation were also upregulated among patients with TRAPS including MAPK14 (2-fold), NFKB1 (1.3-fold), TLR5 (2.4-fold) and MMP9 (2.4-fold) among others. Among genes involved in the TLR signalling pathway, eight genes were upregulated by at least 1.4 -fold in patients with active TRAPS (figure 1A). Of note, IL-1 $\beta$ was upregulated at the gene expression level by 1.8 -fold in patients with TRAPS. Four other genes in the IL-1 immune response signalling pathway were also upregulated by at least 1.4-fold (figure 1B). The upregulation of genes in the apoptosis and survival, endoplasmic reticulum stress response pathway and autophagy pathway maps are shown in figure $1 \mathrm{C}, \mathrm{D}$, respectively.

To identify genes that differed the most between patients with TRAPS and healthy volunteers, a twofold change in gene expression and a corrected $\mathrm{p}$ value threshold of 0.05 were applied. This led to a gene set of the most differentially expressed genes in patients with TRAPS, hereby referred to as the TRAPS disease signature (figure 2, see online supplementary table S2).

\section{Canakinumab alters the expression of genes relevant to TRAPS pathogenesis}

Gene expression profiles in patients with TRAPS were dramatically altered by treatment with canakinumab. Genes meeting a $\geq$ twofold change and $\mathrm{p}$ value cut-off of 0.05 between baseline and day 15 in the canakinumab-treated patients with TRAPS defined a canakinumab treatment signature (figure 3, see online supplementary table S3). Changes in expression of these genes were largely maintained with continued canakinumab treatment through day 113. Many ( $>40 \%$ ) of the canakinumab treatment signature genes (see online supplementary table S2) were also among the TRAPS disease signature genes (see online supplementary table S3), indicating that many genes differentially expressed in patients with active TRAPS compared with healthy volunteers are also differentially expressed postcanakinumab treatment relative to baseline. In fact, $95.9 \%$ of the TRAPS disease signature genes had expression levels move towards that of the healthy volunteers by day 15 of canakinumab treatment (figure 2).

Of note, the disease-causing gene TNFRSF1A had expression levels decreased by 1.4-fold upon canakinumab treatment. As mentioned above, this gene was upregulated by 1.4 -fold in untreated patients with TRAPS compared with healthy volunteers. Likewise, the drug target gene $I L-1 B$ was upregulated in patients with TRAPS by 1.8 -fold relative to healthy volunteers, then downregulated by 1.9 -fold upon canakinumab treatment (figure 4).

In addition, other inflammation-related genes were downregulated after canakinumab treatment. Using genes that changed by at least 1.4-fold, several inflammation-related and innate immune response-related pathways were evident among the differentially expressed genes, including the immune response IL-1 pathway. Notably, MAPK14, the gene encoding the p38 MAP kinase, was among both the TRAPS disease signature genes and canakinumab treatment signature genes; it is found in the IL-1 pathway downstream from the drug target IL-1 $\beta$ (figures $1 \mathrm{~B}$ and 4). Other genes in the IL-1 pathway, such as NFKB1, were also affected by canakinumab treatment, but to a lesser degree.

\section{Changes in neutrophil count are not sufficient to explain the canakinumab treatment signature}

As previously reported, ${ }^{19}$ canakinumab treatment reduces neutrophil counts in whole blood. Since gene expression profiles 
A

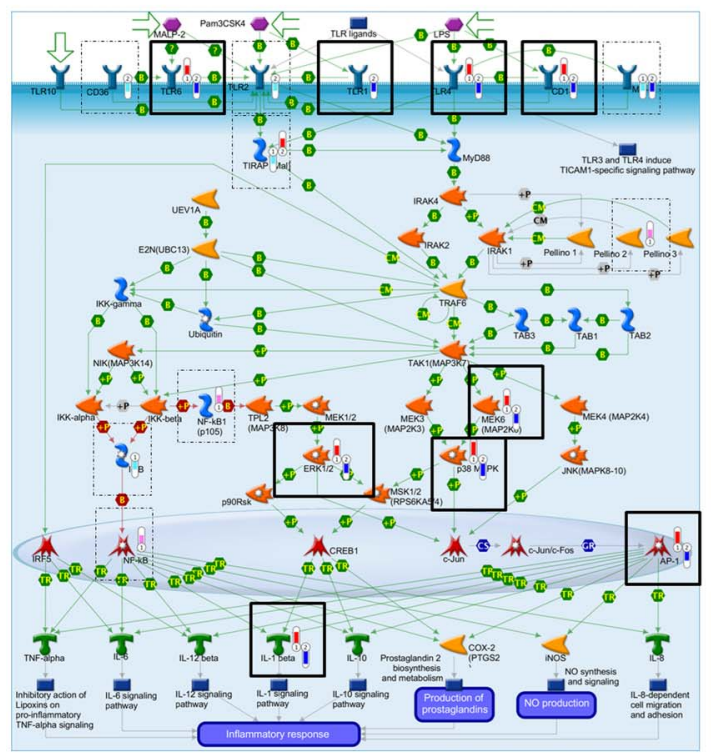

B

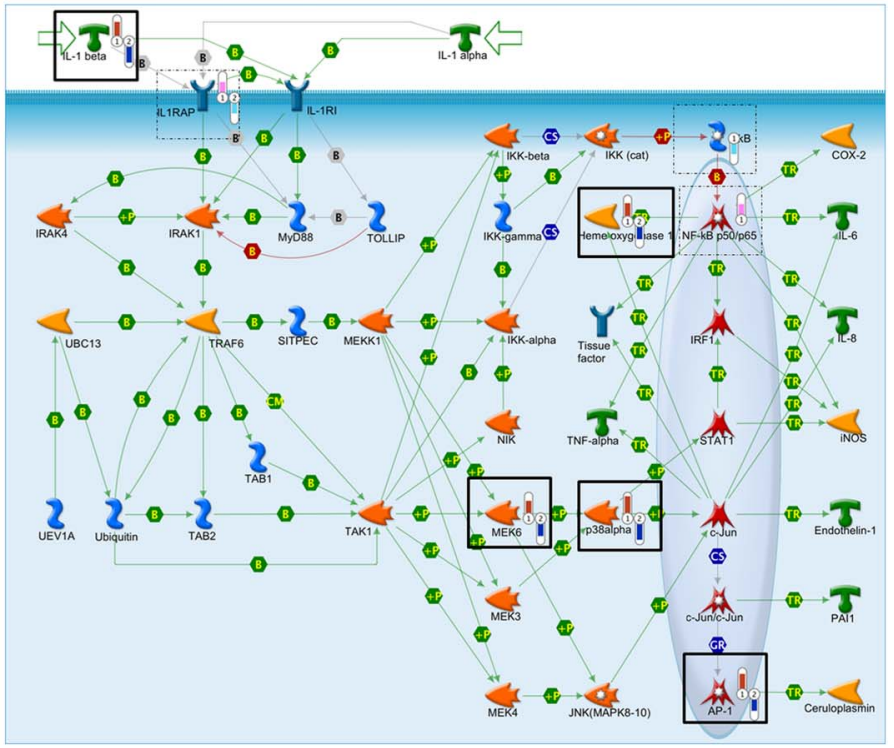

C

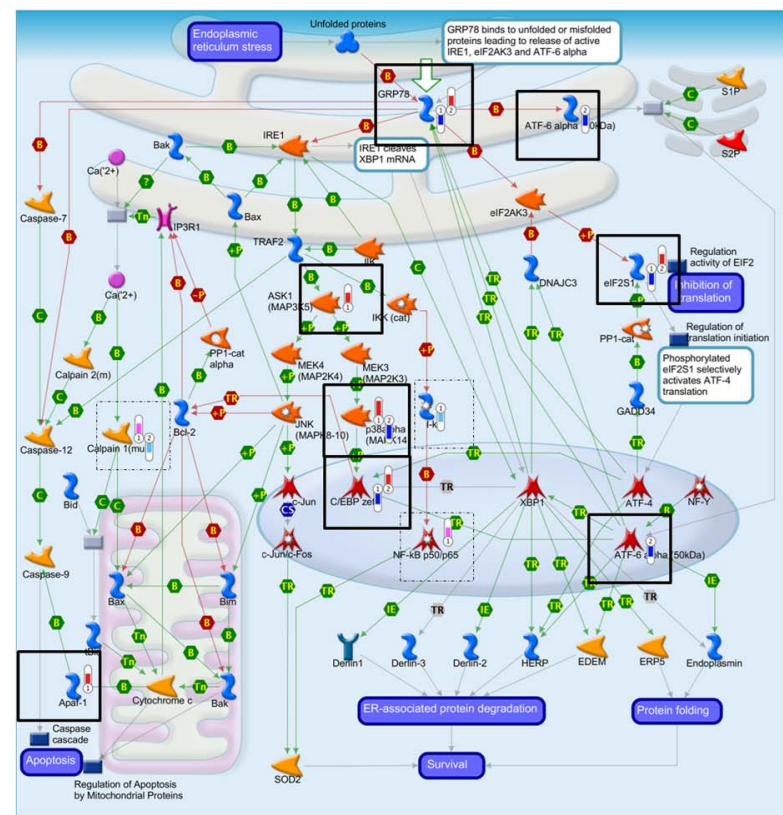

D

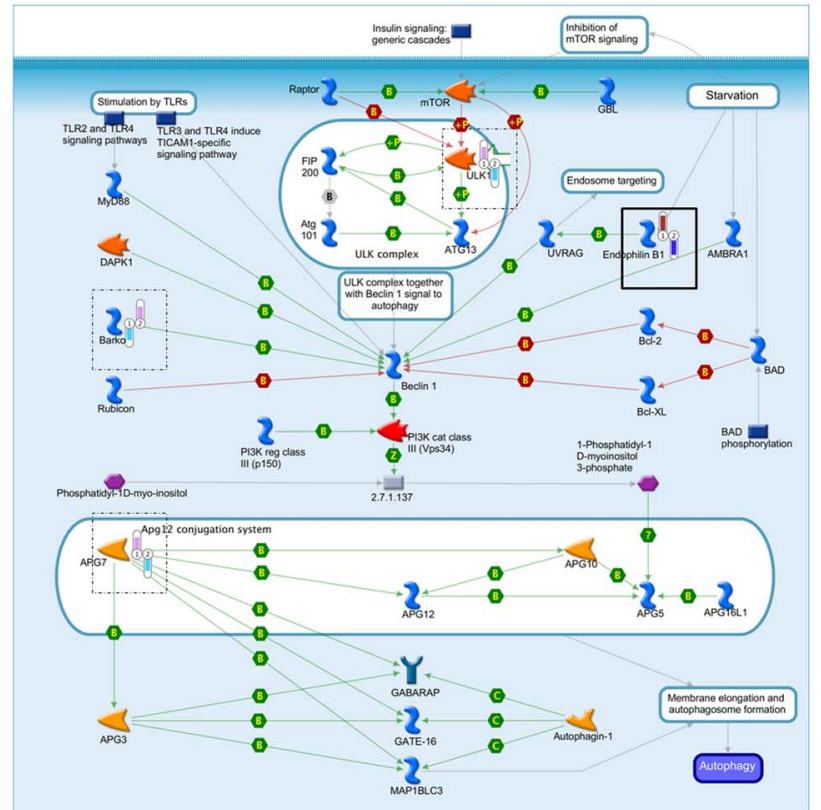

Figure 1 Pathway maps showing changes in gene expression for tumour necrosis factor receptor-associated periodic syndrome (TRAPS) patients compared with healthy volunteers and for patients with TRAPS with canakinumab treatment compared with baseline. (A) Immune response TLR signalling. (B) Immune response IL-1 signalling. (C) Apoptosis and survival: endoplasmic reticulum stress response. (D) Autophagy. Next to each differentially expressed gene, a small icon representing a thermometer can be seen. The thermometers marked as ' 1 ' reflect transcriptional levels at baseline relative to healthy subjects, while the ones marked as ' 2 ' reflect changes post-treatment. Downregulation is indicated by a blue thermometer, while upregulation is marked by a red thermometer, with the level of dysregulation proportional to the length of the colour in the thermometer. Transcripts with a fold change of 1.4 or better are shown in solid black boxes, while those with a fold change between 1.3 and 1.4 are shown in dashed black boxes. Even though many of the fold changes in the pathway maps are lower than 1.4, the directionality of the dysregulation is consistently opposite between patients with TRAPS at baseline versus healthy subjects and in postcanakinumab-treated patients versus precanakinumab-treated patients. This lends support to the interpretation that these transcriptional changes are disease and treatment induced, respectively. In some of the cases, the transcripts are seen to be upregulated in the post-treatment contrast, but there is no thermometer denoted for the disease versus healthy contrast. In many of these cases, the fold change is in the opposite direction, but the absolute value is $<1.3$.

were derived from whole blood, the gene expression changes observed upon canakinumab treatment may have resulted from changes in the proportion of cell types in whole blood and not necessarily from changes in transcriptional regulation. In fact, $65 \%$ of the canakinumab signature genes were correlated $\left(\mathrm{r}^{2}>0.5\right)$ with the neutrophil count. Although neutrophil counts were reduced by an average of approximately 30\% upon treatment with canakinumab, the canakinumab signatures genes exhibited changes in expression of twofold or greater. When the neutrophil count was included in the linear models described above, $52 \%$ of the canakinumab signature genes continued to be differentially expressed post-treatment with $\mathrm{p}<0.05$. 

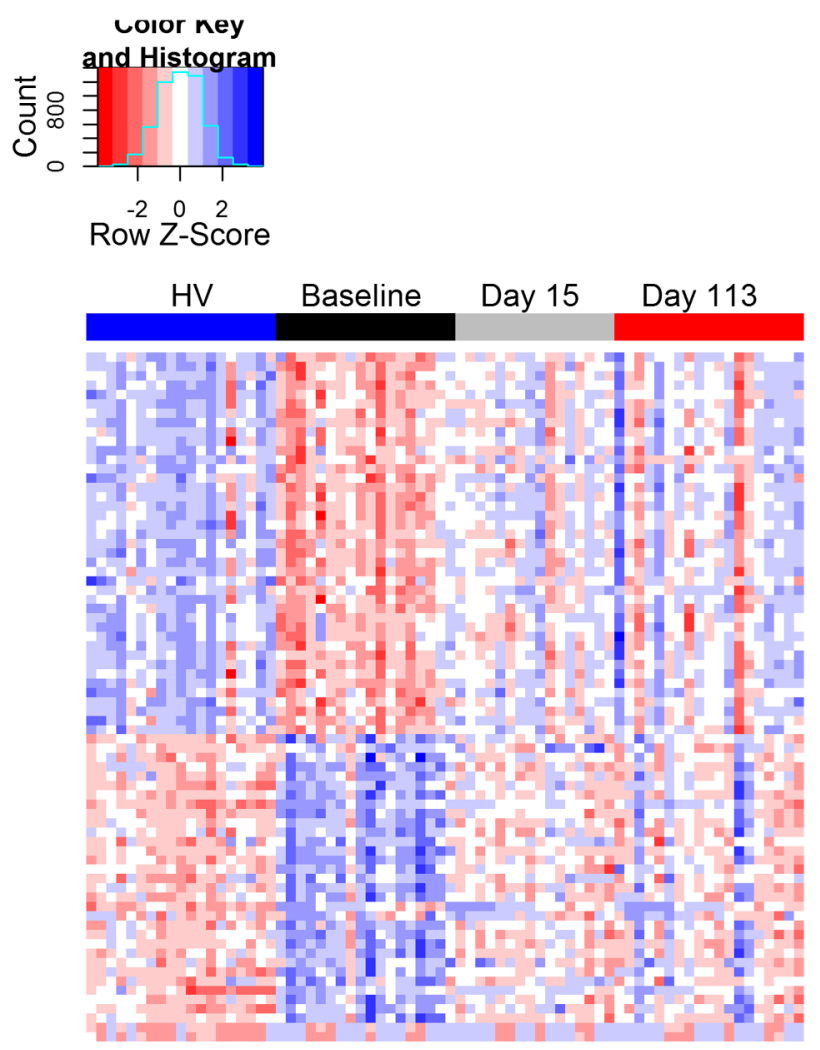

Figure 2 Heatmap of gene expression levels for tumour necrosis factor receptor-associated periodic syndrome (TRAPS) disease signature genes. Genes with a Benjamini-Hochberg corrected $p \leq 0.05$ and an absolute fold change $\geq 2$ between untreated patients with TRAPS at baseline and healthy volunteers define the TRAPS disease signature genes. Each gene is normalised by Z-score across all samples shown. Note how expression levels at both day 15 and day 113 postcanakinumab treatment move towards those seen in healthy volunteers.

\section{DISCUSSION}

TRAPS is a monogenic disease in which the underlying pathogenesis is not yet completely understood. Other groups have previously applied proteomic techniques to elucidate the complexity of intracellular signalling pathways affected by specific TRAPS-associated TNFRSF1A mutations, such as C33Y, and demonstrated increased expression of inflammation-related proteins. ${ }^{20}$ However, it was not clear whether this upregulation could be attributed to protein stability (reduced protein turnover) or to changes at the level of gene expression. Our study focused on gene expression, not proteomics, in a patient group with mixed TNFRSF1A mutations. Our findings build on the literature by showing that gene expression differences are, at least in part, responsible for observed differences in protein expression.

Use of gene expression profiling with comparisons between active disease and healthy volunteers can be a powerful tool in identifying potential molecular mechanisms associated with disease. In addition, the ability to compare expression profiles in patients with active disease and then when they are on treatment with a single cytokine inhibitor allows a unique insight into the molecular effects of canakinumab treatment. Canakinumab altered the gene expression profiles of patients with TRAPS by day 15 , at a time when nearly all patients had achieved complete or near-complete clinical responses. ${ }^{21}$ Notably, the gene expression profiles in patients with TRAPS at
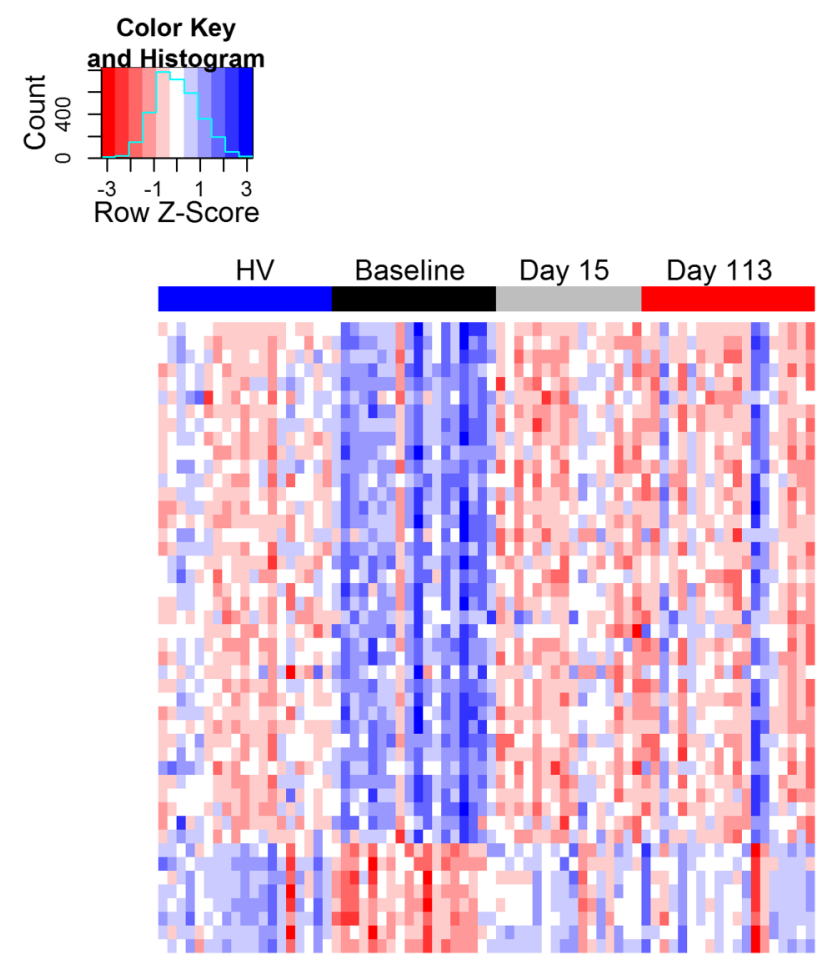

Figure 3 Heatmap of gene expression levels for canakinumab signature genes. Genes with a Benjamini-Hochberg corrected $p \leq 0.05$ and an absolute fold change $\geq 2$ between day 15 post-treatment and baseline in tumour necrosis factor receptor-associated periodic syndrome patients define the canakinumab signature genes. Each gene is normalised by Z-score across all samples shown. Note how expression levels at both day 15 and day 113 postcanakinumab treatment move towards those seen in healthy volunteers.

day 15 more closely resembled those from the age-matched healthy volunteers than those from the patients with TRAPS themselves at baseline. The canakinumab treatment signature genes differentially expressed on day 15 were largely maintained through day 113, suggesting that patients on continued treatment do not generally revert to baseline gene expression levels. Thus, the effect of canakinumab at the molecular level may persist during continued monthly treatment.

We have shown for the first time that the disease-causing gene TNFRSF1A is upregulated at the transcript level in treatmentnaïve patients with active TRAPS compared with healthy volunteers and then downregulated with canakinumab treatment. TNFRSF1A was previously shown to be upregulated in TRAPS at the protein level, with the increase in TNFR1 protein levels attributed to a reduction in its turnover. ${ }^{22}$ Based on the findings of the current study, the increase in the TNFR1 protein may also be due, in part, to an increase in TNFRSF1A transcript levels. These data support the possible role of IL-1 blockade in the downmodulation of one of the major pathogenic factors associated with the development and maintenance of the inflammatory response in TRAPS, namely the intracellular accumulation of the mutated TNFR1 protein.

We have also shown that the drug target gene $I L-1 \beta$ is upregulated at the transcript level in treatment-naïve patients with TRAPS compared with healthy volunteers and then downregulated with canakinumab treatment. Although canakinumab is known to specifically inhibit IL- $1 \beta$ at the protein level, feedback mechanisms in the IL-1 pathway and other innate immune response pathways may also act to downregulate $I L-1 \beta$ at the 

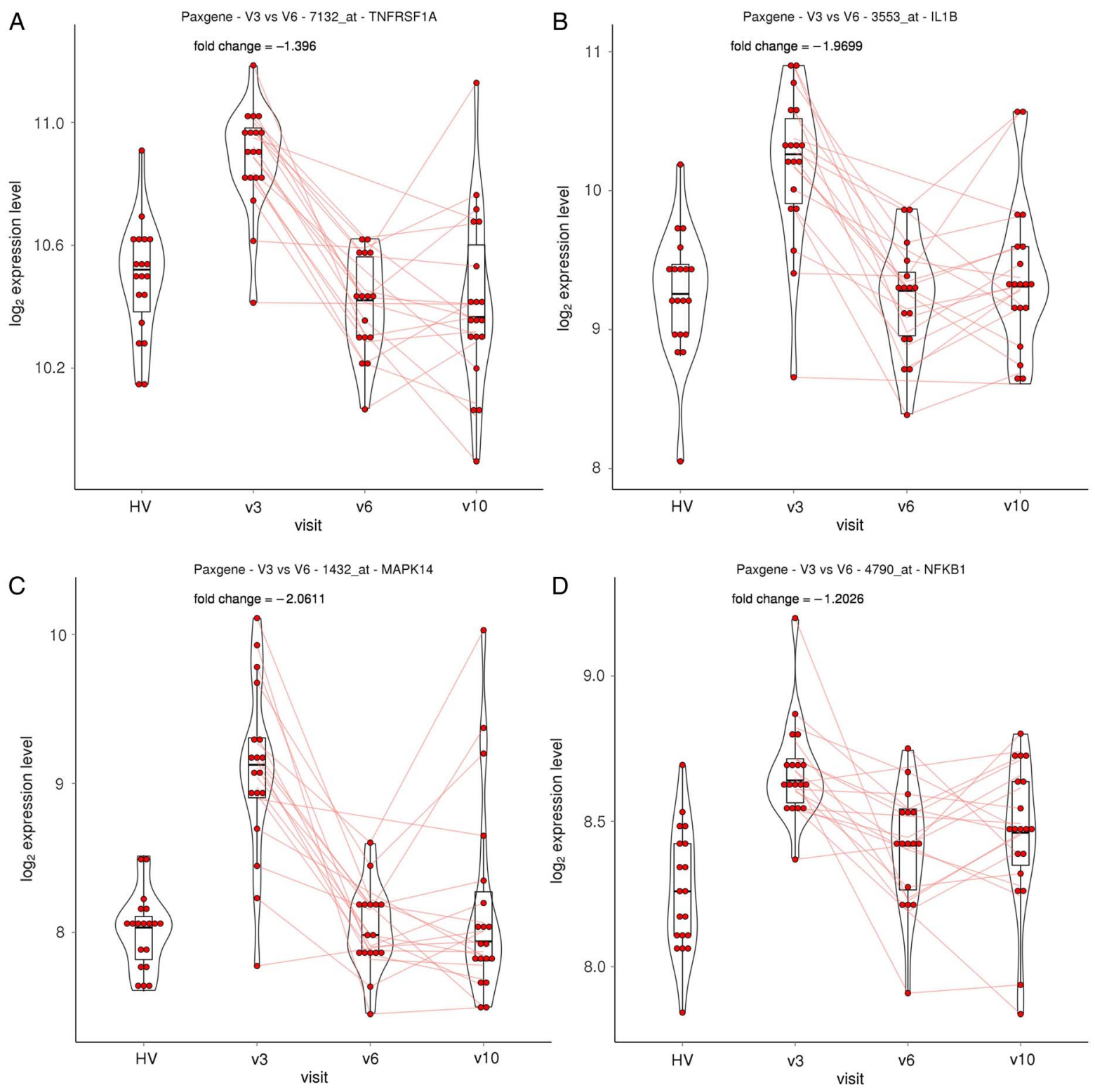

Figure 4 The disease-causing gene (TNFRSF1A, panel A), drug-target gene (IL-1B, panel B) and inflammation-related genes (MAPK14 and NFKB1, panels $C$ and $D$, respectively) are upregulated in tumour necrosis factor receptor-associated periodic syndrome (TRAPS) patients and downregulated upon canakinumab treatment. Lines connect data points originating from the same patient. The figure titles within each panel contain both the Entrez gene IDs (numbers preceding '_at') and the gene symbols. Note how these genes are upregulated at baseline in patients with TRAPS relative to healthy volunteers. Upon treatment at day 15, the expression levels of the genes are reduced to those observed in healthy volunteers and this reduction is maintained through day 113. The fold change between baseline and visit 6 is indicated at the top of each graph.

transcriptional level after treatment with canakinumab. In support of an IL-1 $\beta$ feedback mechanism, in vitro studies have shown that increasing IL-1 protein levels can induce IL-1 $\beta$ expression. ${ }^{23}$ Canakinumab has been shown to disrupt this positive feedback loop in patients with CAPS. ${ }^{16}$ The gene expression data in the current study suggest a similar disruption of the IL-1 $\beta$ feedback loop with canakinumab in patients with TRAPS, as previously suggested by the normalisation of monocyte IL-1 $\beta$ secretion from anakinra-treated patients with TRAPS. ${ }^{8}$

The gene expression and pathway data in the current study indicate that IL-1 blockade with canakinumab treatment increases neutrophil apoptosis and decreases pro-inflammatory signalling. MAPK14, which is downregulated after canakinumab treatment, is present in the IL-1 pathway downstream of the drug target IL-1 $\beta$. The p38 kinase encoded by MAPK14 was activated in a mouse model of TRAPS, resulting in an increased inflammatory response; ${ }^{22}$ further, apoptosis is induced when this family of kinases is inhibited. ${ }^{24}$ Inhibition of MAP kinases may induce apoptosis through inhibition of NF- $\kappa \mathrm{B}$, a negative regulator of apoptosis in the IL-1 pathway and a positive regulator of inflammation. The NF- $\mathrm{BB}$ pathway is upregulated by TRAPS-associated TNFRSF1A mutations. ${ }^{25} 26$ Downregulation of NF- $\kappa \mathrm{B}$ is expected to result in an increase in apoptosis and a concomitant decrease in inflammatory response. In the current study, NF- $\mathrm{BB}$ was upregulated 1.3-fold in patients with TRAPS at baseline compared with healthy volunteers, and downregulated by 1.2 -fold with canakinumab treatment at day 15 compared with baseline.

Patients with TRAPS also appear to have reduced TNF $\alpha$-induced apoptosis in neutrophils, ${ }^{27}$ and the resulting 
accumulation of neutrophils may in turn lead to the accumulation of pro-inflammatory cytokines and thus contribute to TRAPS pathogenesis. ${ }^{1}$ Thus, the reduction in neutrophil counts in patients with TRAPS-presumably reflected in part by MAPK14 and NF- $\mathrm{BB}$ downregulation-may also contribute to the clinical response achieved with canakinumab. However, it is likely that changes in gene expression, in addition to the reduction in neutrophil count, contribute to the improved condition in patients with TRAPS after treatment with canakinumab. Even after controlling for neutrophil count, more than half of the canakinumab treatment signature genes had significant changes in expression levels. Many of these genes are involved in inflammatory signalling and their downregulation would be expected to reduce such signalling.

In previous studies, canakinumab was shown to produce clinical benefit in most patients with systemic juvenile idiopathic arthritis (SJIA). ${ }^{19}$ Interestingly, 39\% of the canakinumab treatment signature genes identified in patients with TRAPS were also differentially expressed in SJIA patients upon treatment (Novartis data on file). Although the filtering criteria and statistical modelling varied by study, the substantial overlap between data sets is representative of the underlying biology of canakinumab treatment. Similar to the TRAPS data, the differentially expressed genes common to both SJIA and TRAPS correlated with reductions in neutrophil counts. However, the reduction in neutrophil counts does not fully explain the downregulation of these genes, leading to the conclusion that the two diseases share transcriptional profiles underlying the biology of canakinumab treatment.

We assessed several pathway maps due to their relevance to TRAPS and to the action of canakinumab. Many of these disease mechanisms were identified initially from studies conducted in monocytes and murine models. ${ }^{4}$ In the pathways examined, there was a notable trend for upregulation of genes at baseline that are relevant to disease pathogenesis and a subsequent downregulation upon canakinumab treatment. For example, several cell surface receptors (eg, TLR1, TLR4, TLR6, CD15) found in the immune response TLR signalling pathway were perturbed in patients with TRAPS relative to healthy volunteers, suggesting that they contribute to the inflammatory response in TRAPS (see figure 1A). Several key players in this pathway were upregulated at baseline and downregulated after canakinumab treatment, consistent with the inhibition of IL-1 $\beta$ by canakinumab. Similarly, in the immune response IL-1 signalling map, several nodes (eg, IL-1, MAPK14) were evident reflecting transcriptional upregulation in patients with TRAPS compared with the healthy volunteers, and subsequently downregulation after canakinumab treatment compared with baseline (see figure 1B). Because many of the same genes in these pathways are both upregulated in patients with TRAPS and then downregulated by canakinumab, it lends confidence that these pathways are involved in both TRAPS disease and treatment modulation. However, it is also possible that TLR4 upregulation in TRAPS and subsequent downregulation with canakinumab likely reflects changes in cell populations. Instead, the most important mechanism associated with the downregulation of IL-1 $\beta$ production may be the downregulation of IL-1 $\beta$ itself, since there is evidence that IL-1 acts as one of the major inducers of IL-1 itself. ${ }^{28}{ }^{29}$ Although IL- $1 \alpha$ is also known to stimulate IL-1 $\beta$ production, ${ }^{20}$ the use of specific probes for these cytokines suggests that IL-1 $\beta$ is the important driver of IL-1 $\beta$ production in TRAPS.

In the apoptosis and survival: endoplasmic reticulum stress response pathway map, upregulation of GRP78 and downregulation of MAPK14 after canakinumab treatment were observed, leading to neutrophil apoptosis and to the endoplasmic reticulum-associated protein degradation of misfolded proteins (see figure 1C). In the autophagy map, the largest transcriptional changes were seen for endophilin B1 and in TLR signalling (see figure 1D). The autophagy process is thought to be defective in the presence of excessive intracellular amounts of mutant TNFR1 protein, leading to the induction of NF- $\mathrm{B}$ activity and IL-1 $\beta$ oversecretion. ${ }^{8}$ However, the number of transcriptionally affected genes relevant to autophagy and inflammasome-associated gene processes in the two contrasts (patients with TRAPS vs healthy volunteers, and patients with TRAPS postcanakinumab vs baseline) were not sufficient to draw any conclusions about their relevance in our cohort. Mitochondrial reactive oxygen species have been identified as a key driver of inflammation in TRAPS. ${ }^{30}$ We examined pathway maps for oxidative phosphorylation and superoxide production, and observed changes for eight genes in the latter map similar to those shown in the maps in figure 1, namely an increase in expression in patients with TRAPS at baseline compared with healthy volunteers and a decrease in expression upon canakinumab treatment.

It is important to recognise that gene expression does not necessarily correlate with protein production, and therefore further studies would be useful to determine the pathogenic relevance of the gene expression changes reported herein. Potentially relevant studies include measuring the protein products themselves, or performing in vitro gene knockout or knockin studies. Additionally, other methods for measuring the implicated signature genes appear warranted to further understand mechanisms important in the disease and canakinumab action.

Taken together, the present findings support a model in which IL-1 $\beta$ inhibition by canakinumab downregulates genes in the IL-1 signalling and other innate immunity pathways that are overexpressed in TRAPS. Treatment with canakinumab downregulates both the disease-causing and drug-target genes resulting in the downregulation of inflammatory responses. Treatment with canakinumab also induces apoptosis of neutrophils. Together, the decrease in pro-inflammatory signalling and the reduction in neutrophil counts most likely contribute to the changes seen in patients with TRAPS at the transcriptional level during canakinumab treatment. In turn, these molecular changes likely contribute to the clinical improvement seen in these patients, suggesting that canakinumab produces diseasemodifying effects in TRAPS.

Acknowledgements We thank the participating patients and investigators. This study was supported by Novartis Pharma AG. We thank Elise Hellinger and BioScience Communications, and Katia de Souza, Kathleen Graham Lomax, MD, and Uwe Machein, MD for providing editorial support on behalf of Novartis Pharma AG. We would also like to thank Lukas Baeriswyl, who was responsible for the quality control of the microarray data.

Contributors All authors contributed to the data collection and/or writing of the manuscript, and have thoroughly reviewed and approved of the content.

Funding This study was sponsored and funded by Novartis Pharma AG.

Competing interests KA: employee of Novartis Pharmaceuticals. MC: received speaker fees and served as a consultant for Novartis and SOBI. MG: received speaker fees and served as a consultant for Novartis and SOBI, and has received unrestricted grants for the Eurofever Registry from Novartis and SOBI. NH: employee of Novartis Institutes for Biomedical Research. $\mathrm{HL}$ : received speaker fees and served as a consultant for Novartis and SOBI. ML: employee of Novartis Institutes for Biomedical Research. NN: employee of Novartis Institutes for Biomedical Research. LO: received speaker fees and served as a consultant for Novartis. SS-S: employee of Novartis Institutes for Biomedical Research. RT: employee of Novartis Institutes for Biomedical Research. 
Ethics approval The study was conducted according to the ethical principles of the Declaration of Helsinki.Independent Ethics Committee (IEC) or Institutional Review Board (IRB): The study protocol and all amendments were reviewed by the IEC or IRB for each centre: Clinical Research Ethics Committee, Merlin Park Hospital, Galway, Ireland; National Research Ethics Service, Southampton and South West Hampshire REC(B), Berkshire, UK; Comitato Di Bioetica Dell' IRCCS Istituto Giannina Gaslini Di Genova, Genova, Italy; Comitato Di Bioetica Della Fondazione IRCCS Policlinico San Matteo Di Pavia, Pavia, Italy; Comitato Etico Azienda ospedaliera Spedali Civili Di Brescia, Brescia, Italy; Comitato Etico Dell'ASL 1 Di Agrigento, Agrigento, Italy.

Provenance and peer review Not commissioned; externally peer reviewed.

Open Access This is an Open Access article distributed in accordance with the Creative Commons Attribution Non Commercial (CC BY-NC 4.0) license, which permits others to distribute, remix, adapt, build upon this work non-commercially, and license their derivative works on different terms, provided the original work is properly cited and the use is non-commercial. See: http://creativecommons.org/ licenses/by-nc/4.0/

\section{REFERENCES}

1 Kimberley FC, Lobito AA, Siegel RM, et al. Falling into TRAPS - receptor misfolding in the TNF receptor 1-associated periodic fever syndrome. Arthritis Res Ther 2007;9:217.

2 Ter Haar N, Lachmann $\mathrm{H}$, Özen $\mathrm{S}$, et al. Treatment of autoinflammatory diseases: results from the Eurofever Registry and a literature review. Ann Rheum Dis 2013;72:678-85

3 McDermott MF, Aksentijevich I, Galon J, et al. Germline mutations in the extracellular domains of the $55 \mathrm{kDa}$ TNF receptor, TNFR1, define a family of dominantly inherited autoinflammatory syndromes. Cell 1999;97:133-44.

4 Hull KM, Drewe E, Aksentijevich I, et al. The TNF receptor-associated periodic syndrome (TRAPS): emerging concepts of an autoinflammatory disorder. Medicine (Baltimore) 2002;81:349-68.

5 Vitale A, Rigante D, Lucherini OM, et al. Biological treatments: new weapons in the management of monogenic autoinflammatory disorders. Mediators Inflamm 2013;2013:939847.

6 Lachmann HJ, Papa R, Gerhold K, et al. The phenotype of TNF receptor-associated autoinflammatory syndrome (TRAPS) at presentation: a series of 158 cases from the Eurofever/EUROTRAPS international registry. Ann Rheum Dis 2014;73:2160-7.

7 Gattorno M, Martini A. Beyond the NLRP3 inflammasome: autoinflammatory diseases reach adolescence. Arthritis Rheum 2013;65:1137-47.

8 Bachetti T, Chiesa S, Castagnola P, et al. Autophagy contributes to inflammation in patients with TNFR-associated periodic syndrome (TRAPS). Ann Rheum Dis 2013;72:1044-52.

9 Dinarello CA. Biologic basis for interleukin-1 in disease. Blood 1996;87:2095-147.

10 Dinarello CA. Blocking IL-1 in systemic inflammation. J Exp Med 2005;201:1355-9.

11 Lobito AA, Kimberley FC, Muppidi JR, et al. Abnormal disulfide-linked oligomerization results in ER retention and altered signaling by TNFR1 mutants in TNFR1-associated periodic fever syndrome (TRAPS). Blood 2006;108:1320-7.

12 Dickie LJ, Aziz AM, Savic S, et al. Involvement of X-box binding protein 1 and reactive oxygen species pathways in the pathogenesis of tumour necrosis factor receptor-associated periodic syndrome. Ann Rheum Dis 2012;71:2035-43.
13 Greco E, Aita A, Galozzi P, et al. The novel S59P mutation in the TNFRSF1A gene identified in an adult onset TNF receptor associated periodic syndrome (TRAPS) constitutively activates NF- $\mathrm{KB}$ pathway. Arthritis Res Ther 2015;17:93.

14 Gattorno M, Pelagatti MA, Meini A, et al. Persistent efficacy of anakinra in patients with tumor necrosis factor receptor-associated periodic syndrome. Arthritis Rheum 2008;58:1516-20.

15 Obici L, Meini A, Cattalini M, et al. Favourable and sustained response to anakinra in tumour necrosis factor receptor-associated periodic syndrome (TRAPS) with or without AA amyloidosis. Ann Rheum Dis 2011;70:1511-12.

16 Lachmann $H J$, Lowe $P$, Felix SD, et al. In vivo regulation of interleukin $1 \beta$ in patients with cryopyrin-associated periodic syndromes. J Exp Med 2009;206:1029-36.

17 Dhimolea E. Canakinumab. mAbs 2010;2:3-13.

18 NCT01242813. An open-label, multicenter, efficacy and safety study of 4-month canakinumab treatment with 6-month follow-up in patients with active recurrent or chronic TNF-receptor associated periodic syndrome (TRAPS). https://clinicaltrials.gov/ ct2/show/NCT01242813?term=NCT01242813\&rank=1 (accessed 16 Aug 2015).

19 Ruperto $\mathrm{N}$, Brunner HI, Quartier $\mathrm{P}$, et al. Two randomized trials of canakinumab in systemic juvenile idiopathic arthritis. N Engl J Med 2012;367:2396-406.

20 Negm OH, Mannsperger HA, McDermott EM, et al. A pro-inflammatory signalome is constitutively activated by C33Y mutant TNF receptor 1 in TNF receptorassociated periodic syndrome (TRAPS). Eur J Immunol 2014;44:2096-110.

21 Gattorno M, Obici L, Meini A, et al. Efficacy and safety of canakinumab in patients with TNF receptor associated periodic syndrome. Ann Rheum Dis 2012;71(Suppl 3):289.

22 Simon A, Park H, Maddipati R, et al. Concerted action of wild-type and mutant TNF receptors enhances inflammation in TNF receptor 1-associated periodic fever syndrome. Proc Natl Acad Sci USA 2010;107:9801-6.

23 Dinarello CA, Ikejima T, Warner SJC, et al. Interleukin 1 induces interleukin 1. I. Induction of circulating interleukin 1 in rabbits in vivo and in human mononuclear cells in vitro. J Immunol 1987;139:1902-10.

24 Kamata $\mathrm{H}$, Honda $\mathrm{S}$, Maeda $\mathrm{S}$, et al. Reactive oxygen species promote TNF $\alpha$-induced death and sustained JNK activation by inhibiting MAP kinase phosphatases. Cell 2005;120:649-61.

25 Nedjai B, Hitman GA, Yousaf N, et al. Abnormal tumor necrosis factor receptor I cell surface expression and NF- $\mathrm{KB}$ activation in tumor necrosis factor receptor-associated period syndrome. Arthritis Rheum 2008;58:273-83.

26 Churchman SM, Church LD, Savic S, et al. A novel TNFRSF1A splice mutation associated with increased nuclear factor kappaB (NF- $\kappa \mathrm{B})$ transcription factor activation in patients with tumour necrosis factor receptor associated periodic syndrome (TRAPS). Ann Rheum Dis 2008;67:1589-95.

27 D'Osualdo A, Ferlito F, Prigione I, et al. Neutrophils from patients with TNFRSF1A mutations display resistance to tumor necrosis factor-induced apoptosis. Arthritis Rheum 2006;54:998-1008.

28 Goldbach-Mansky R, Kastner DL. Autoinflammation: the prominent role of IL-1 in monogenic autoinflammatory diseases and implications for common illnesses. J Allergy Clin Immunol 2009;124:1141-9.

29 Gattorno M, Tassi S, Carta S, et al. Pattern of interleukin-1 $\beta$ secretion in response to lipopolysaccharide and ATP before and after interleukin-1 blockade in patients with CIAS1 mutations. Arthritis Rheum 2007;56:3138-48.

30 Bulua AC, Simon A, Maddipati $R$, et al. Mitochondrial reactive oxygen species promote production of proinflammatory cytokines and are elevated in TNFR1-associated periodic syndrome (TRAPS). J Exp Med 2011;208:519-33. 